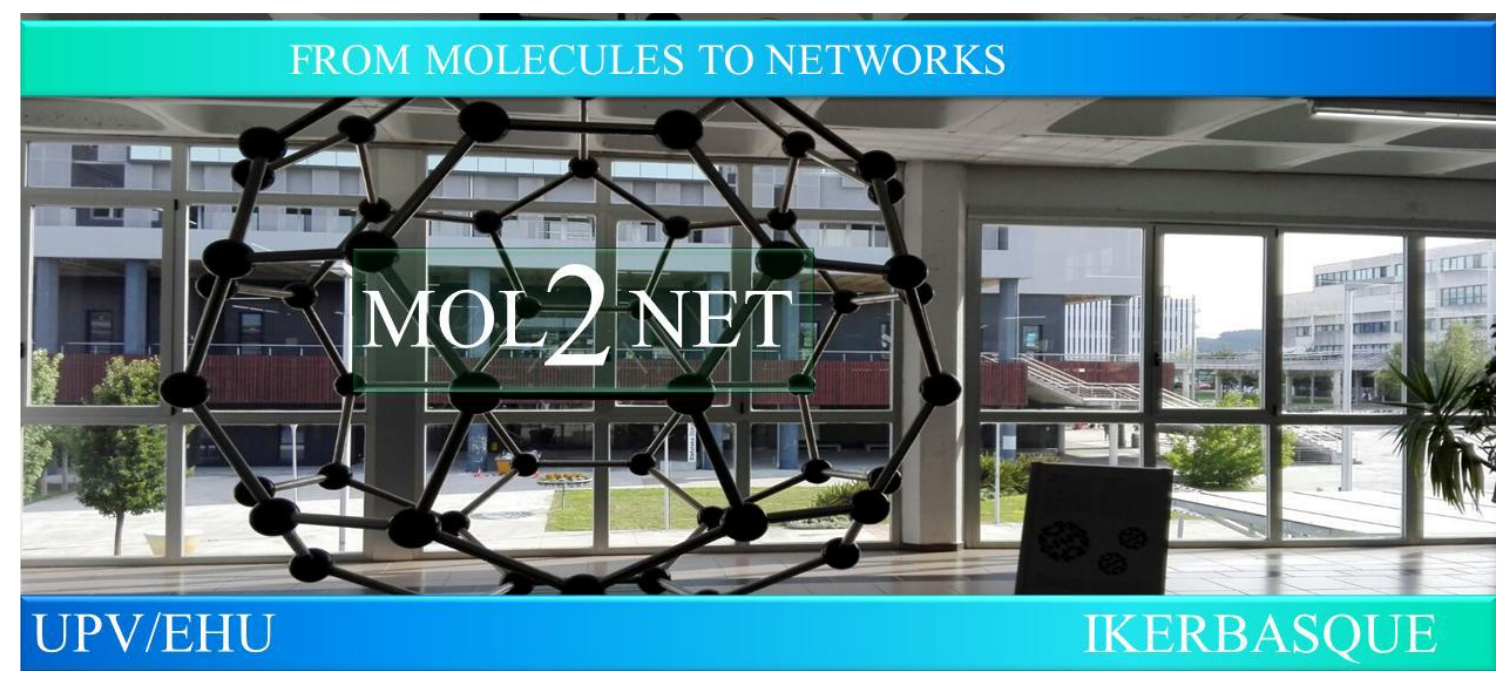

During the last few years, we have learned a lot about diabetes, especially with the passage of time and biotechnological advances, we have seen that not only exist type I or type II diabetes exist, but it is made up of many more types. In the current study, information has been collected from different types of patients and it was deduced that the results in type II diabetes could be improved by identifying what type of treatment should be given to each patient so that they obtain the greatest benefit from the control treatment personalized glycemic from the machine learning model.

The key feature was to identify an improvement and maintenance of glycosylated hemoglobin $(\mathrm{HbA} 1 \mathrm{c})$ in patients below $7 \%$ using machine learning when analysing data from combination therapy clinical trials. The trials were focused on phase 3, randomized, double-blind, parallel-group studies treated with a combination of empagliflozin and linagliptin against patients receiving metformin alone. The evaluation of the treatments of the two patient groups was written in detail by the descriptive analysis of the univariate associations between the $\mathrm{HbA1c}$ target categories and each baseline characteristic. Upon finalizing the results of the descriptive analysis, machine learning tools such as the classification tree and random forest methods were used to estimate and predict target categories based on patient characteristics.

Maintaining the HbA1c study target protein was shown to be associated with lower baseline $\mathrm{HbA} 1 \mathrm{c}$ protein and fasting plasma glucose. With the learning method we saw that the strongest indicators to achieve glycemic maintenance are achieved precisely with the HbA1c protein and fasting plasma glucose. In the experiment, it was other covariates such as body weight, waist circumference, and blood pressure that did not contribute to the final result of the study. 
a Empagliflozin/linagliptin
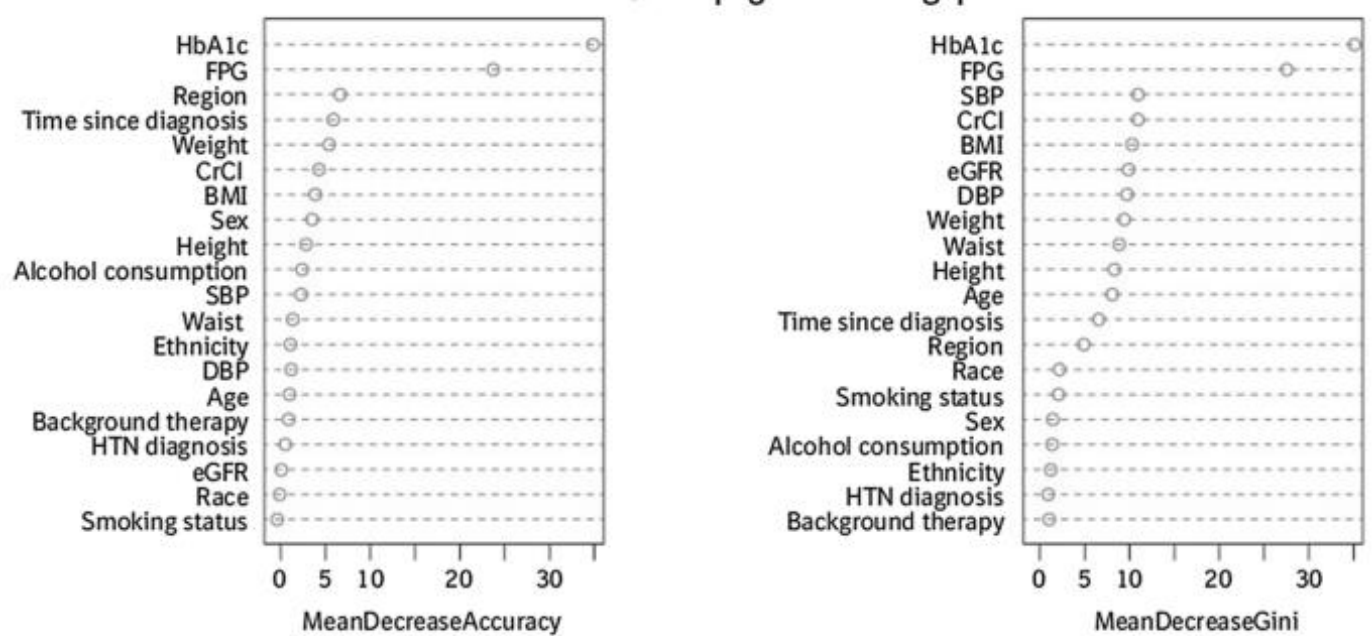

\section{b Empagliflozin}
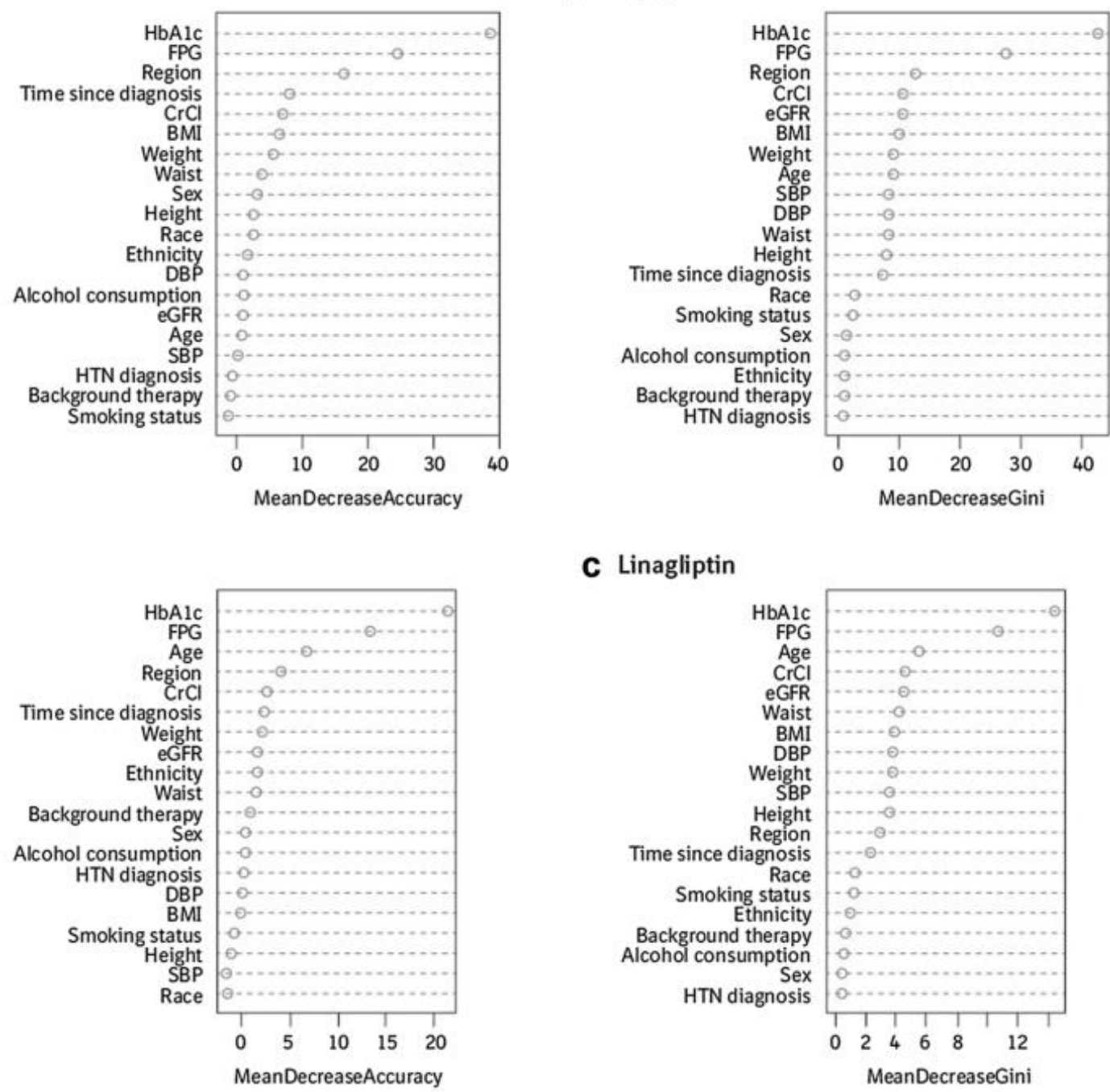

\section{Linagliptin}

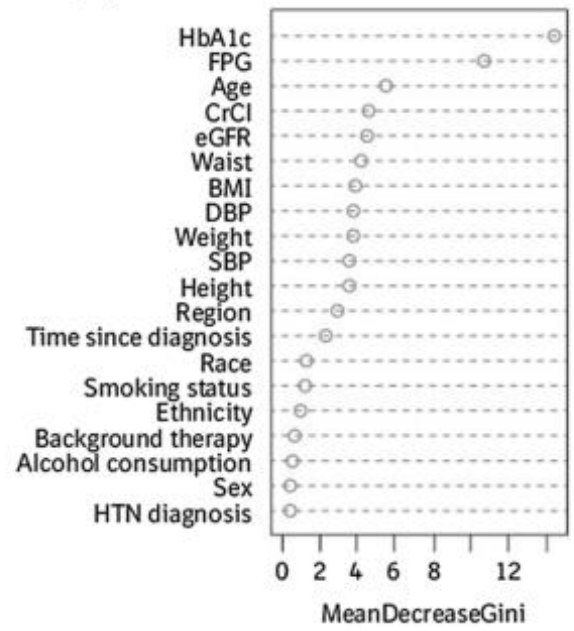

Figure 1. Importance of baseline variables in the random forest analysis for a single pill combination of empagliflozin / linagliptin, empagliflozin b, and linagliptin c 
With this study, it could be concluded that using a new machine learning model we could introduce new analysis models to be able to process a large amount of traditional data and be able to extract information such as that of the strongest preacher of the achievement of glycemic control. And above all, these predictions made by the new analyzes could make it easier for us to make clinical decisions in order to have a more effective and personalized treatment for each patient according to their disease status.

- (1): Del Parigi, A., Tang, W., Liu, D. et al. Machine Learning to Identify Predictors of Glycemic Control in Type 2 Diabetes: An Analysis of Target HbA1c Reduction Using Empagliflozin/Linagliptin Data. Pharm Med 33, 209-217 (2019).

https://doi.org/10.1007/s40290-019-00281-4 